\title{
reCHERches
}

\section{Le mensonge et ses doubles}

Isabelle Smadja

\section{(2) OpenEdition}

Journals

Édition électronique

URL : https://journals.openedition.org/cher/377

DOI : 10.4000/cher.377

ISSN : 2803-5992

\section{Éditeur}

Presses universitaires de Strasbourg

\section{Édition imprimée}

Date de publication : 19 novembre 2020

Pagination : 135-144

ISBN : 979-10-344-0071-3

ISSN : 1968-035X

\section{Référence électronique}

Isabelle Smadja, "Le mensonge et ses doubles », reCHERches [En ligne], 25 | 2020, mis en ligne le 01 octobre 2021, consulté le 21 novembre 2021. URL : http://journals.openedition.org/cher/377 ; DOI : https://doi.org/10.4000/cher.377 


\title{
Le mensonge et ses doubles
}

\author{
ISABELLE SMADJA ${ }^{1}$
}

$\mathrm{L}$ e théâtre, c'est une évidence, est un art du mensonge: mensonge de l'acteur qui pleure "pour rien, pour Hécube! Qu'est-ce qu'Hécube pour lui?» (Shakespeare 1603: 54), mensonge du spectateur qui se berce de l'illusion que l'acteur ne ment pas, mensonge du dramaturge qui veut nous faire croire qu'à l'hameçon de la fiction, nous pourrions attraper, tel Hamlet, la «carpe vérité.» Dès lors, mentir au théâtre serait-ce rétablir la vérité en nous faisant prendre conscience de la duperie du théâtre? Certes non. Car, pour paraphraser Pascal nous prévenant contre les fourberies de l'imagination, «le théâtre, maître d'erreur et de fausseté, est d'autant plus fourbe qu'il ne ment pas toujours. Car il serait règle infaillible de vérité, s'il l'était infaillible de la fausseté.» C'est à observer cet usage flottant du mensonge, maître tout à la fois d'erreur et de vérité, que nous consacrerons cette étude des pièces de deux dramaturges argentins nés peu après 1960, Claudio Tolcachir et Rodrigo Garcia. Nous montrerons comment ils usent de la relation complexe entre mensonge et vérité avec des objectifs antithétiques. Car si, pour Garcia, il faut mentir au théâtre pour rétablir la vérité du théâtre qui n'est que mensonge, pour Tolcachir en revanche, il faut montrer les personnages en train de mentir pour les contraindre à révéler la vérité qu'ils voulaient dissimuler: le dévoilement du mensonge dans lequel les personnages se complaisaient, permettra ainsi aux spectateurs d'être mis en présence de l'intimité des êtres, c'est-à-dire de leur vérité. Si donc, dans le théâtre de Tolcachir, mentir au théâtre provoque un effet de réel qui redouble, paradoxalement, l'effet de vérité, l'objectif de Garcia paraît être d'obliger à une distanciation par le rappel systématique que tout n'est que mensonge.

\section{«Au commencement était le mensonge...» (Py 2011:3)}

Dans nombre de ses pièces, Garcia s'évertue à créer systématiquement une confusion entre vérité et mensonge. L'objectif pourrait être d'agresser les

1 Agrégée, docteure, professeure de philosophie, auteur de La folie au théâtre (PUF). 
spectateurs dans leur libido sciendi, leur pulsion de savoir: incapables de décider du vrai et du faux, ils sont sciemment écartés du processus de dévoilement d'une vérité que pourtant le texte de Garcia, très politique, semble leur destiner. Car les affirmations des personnages que Garcia met en scène oscillent tellement entre le «parler-vrai» et l'ironie mensongère, que nous ne savons plus distinguer le vrai du faux. Le texte de Garcia abonde de propos qui, à peine énoncés, sont aussitôt contredits. Dans Jardinage humain, où l'humour le dispute constamment avec l'ironie grinçante, un personnage anonyme répond à son interlocuteur imaginaire: "Pourquoi je traite mal les gens? Parce que je traite bien mon chien. Ce n'est pas vrai. J'ai maltraité mon chien un paquet d'années, et en même temps j'ai maltraité les gens. " (Garcia 2003: 22-23). Puis, après plusieurs remarques similaires, le dramaturge ajoute à notre trouble en affirmant: «Tu devras bien un jour raconter la vérité à quelqu'un.» (Garcia 2003 : 26) Mais quel objectif poursuit donc Garcia pour nous contraindre ainsi à admettre que rien de ce qui fut dit ni ne sera dit dans cette pièce n'est vrai? En usant et abusant du mensonge et de la provocation, Garcia nous refuse le contrat implicite qui nous lie ordinairement au spectacle et qui nous permet de nous bercer de l'illusion que ce qui est affirmé sur la scène est vrai. C'est le pacte même entre l'auteur et son public que Garcia veut faire exploser. Dans L'Effet personnage dans le roman, Vincent Jouve rappelle le contrat que tout spectateur assume dès qu'il s'introduit dans une fiction:

Il semble que, dans la fiction, soit en jeu une forme de "croire" particulière, qui repose sur la confiance dans les renseignements apportés sur scène. En vertu du pacte implicite qui nous lie à la fiction, nous considérons que ce que le personnage raconte est vrai; et en vertu d'un effet de glissement nous pensons comme vrai non seulement ce qu'il dit des actions ou des situations, mais aussi ce qu'il énonce comme jugement ou comme interprétation (Jouve 1992: 202).

Or, comme pour mieux nous déstabiliser, Garcia nous empêche d'accorder la moindre confiance en la parole du personnage: le mensonge avoué, assumé, est alors clamé comme un cri de guerre contre le public, parce que «tout était faux et merdique. Tout faux. Tout merdique»(Garcia 2011: 53), ou encore parce que "vous êtes tous des porcs et que vous faites tous des choses horribles chez vous quand personne ne vous voit» (Garcia 11/2003 : 25). Pour autant, Garcia ne se satisfait pas du simple mensonge: il use de toutes les facettes du discours faux et nous promène constamment entre l'ironie et le cynisme, le mensonge et la provocation et... une vérité amère. Car c'est bien de vérité que Garcia nous gorge lorsque, par exemple, il nous rappelle que nous préférons que les autres aillent mal pour nous sentir mieux, mais cette vérité est tellement cynique et excessive, qu'elle en devient presque mensongère:

J'ai téléphoné à ma famille qui va toujours mal pour les entendre me dire "on va mal, très mal.” Je venais de me séparer et j'allais vraiment mal. C’est pour ça que j'avais appelé mes parents: pour parler à quelqu'un qui va encore plus mal que moi, pour ne pas me sentir le dernier des malheureux. Mais mon père décroche et me dit: "on va très bien, très très bien." Quelle bande d'enfoirés... (Garcia 2003:31) 
Mais ici qui ment le mieux ou le plus? Le père affirmant qu'ils vont très bien, comme pour faire enrager son fils? Le fils se présentant probablement comme plus cynique qu'il n'est? L'acteur jouant le fils cynique? Ou le dramaturge accentuant le cynisme des uns et des autres pour mieux asseoir son statut de dramaturge provocateur? Dans cet imbroglio de mensonges, ne demeure qu'une question: qui croire ici sinon personne? Qui croire lorsque l'hypocrisie sociale n'est pas le fait uniquement des personnages jouant sur la grande scène du monde, mais également du dramaturge se plaisant à accentuer une telle hypocrisie? Qu'il faille être si désabusé sur la nature humaine qu'il ne nous reste plus rien à croire, telle est bien la leçon qu'Angélica Liddell, autre dramaturge révoltée de la modernité, veut nous inculquer dans Maudit soit l'homme qui se confie à l'homme:

Le monde est juste une organisation de mensonges récompensés. [...] Il y a des gens qui disent "je t'aime" seulement quand ils peuvent obtenir de la graisse en échange. [...] Ne crois personne. [...] Mieux vaut entrer dans une épicerie chinoise et demander: il reste du pain? Oui. C'est combien? Soixante centimes. C'est parfois les seules phrases sincères qu'on peut entendre de la journée (Liddell 2011: 14-15).

Si Garcia compose avec toutes les variantes du mensonge, c'est qu'il est conscient de ce dont nous prévient Jankélévitch: «La vérité à laquelle l'ironiste nous convie, est une vérité trempée dans le jeu du contraire avec son contraire. C'est donc l'ironie qui est réellement ouverte; et c'est le mensonge qui est clos. De là l'aspect lourdement médiocre du mensonge» (Jankelevitch 1979: 61).

Et c'est bien pour éviter la lourde médiocrité du simple mensonge, que Garcia nous procure quelques-uns des échanges les plus savoureux entre ses personnages: "Patricia: Parlons de l'éducation des enfants et des chiens. Carlos: L'éducation des enfants et des chiens est à peu près la même: il faut les frapper»(Garcia 2002: 71). Bref, il y a de l'ironie chez Garcia, de cette ironie qui veut éveiller les consciences, au lieu de nous bercer dans un discours lénifiant. Comparant l'ironie et le mensonge, Jankelevitch précise: "L'ironie ne veut pas être crue, elle veut être comprise: [...] elle sollicite l'intellection et nous dit: "Complétez vous-même, jugez par vous-même!" Le mensonge, à l'inverse, exploitant notre tendance naturelle à croire, est un abus de confiance.» (Jankelevitch 1979: 61-64).

De fait, Garcia ne veut pas être cru et c'est pourquoi il clame haut et fort, dans chacune de ces pièces, qu'il «ment». En revanche, il veut être compris, espérant une prise de conscience de l'immoralité de la position bourgeoise, dont son théâtre paradoxalement est une composante. Pourtant, ne peut-on creuser plus avant? Car s'il y a bien de l'ironie, n'y-a-t-il pas aussi du mensonge dans l'ironie dont il use et dans la misanthropie qu'il affecte d'avoir? Quelle est par exemple la véracité de l'appel à la révolution violente qu'il affecte d'adopter? Pense-t-il vraiment qu'il faut leur «faire sauter la gueule pour qu'ils comprennent ces salopards » comme il s'en vante à maintes reprises? 
Il faut essayer de se faufiler dans leurs maisons. Tu parles! Il n'y arrive pas les pauvres! Ils sont crevés, ils griffonnent sur les murs, insultent! Avec des insultes, on n'arrive nulle part, pauvres lavettes! Il faut escalader! Tu parles! Ils ont de tout les salopards! Dobermans, rottweilers, pittbuls, vigiles/ Ils les ont sortis d'où les chiens? Puisque tout vient d'exploser?/ Et à la fin advient ce qui doit advenir / ceux qui ont le blé ouvrent la porte de leurs maisons / et ils proposent des accords / et ils fixent le prix / [...] Et la populace se calme / et ils se remettent à travailler avec les mêmes salauds (Garcia 11/2003: 32).

D'un certain point de vue, l'indignation qui s'exprime ici est sincère, et correspond probablement à une des composantes de la pensée de Garcia. Mais est-ce si simple? Du fait même de la position qu'il occupe à l'intérieur d'un théâtre subventionné par un système de profit, la décision de «leur faire sauter la gueule, à ces salopards", se transforme en son contraire: en un discours mensonger. Mensonge d'abord, en ce qu'il affirme le contraire de ce qu'il propose: au moment même où il affirme "vous devriez en baver salopards / vingt générations de gagnants qui en foutent pas une rame» (Garcia 11/2003: 33), il propose à ces mêmes "salopards" une soirée en fauteuil où il faut l'avouer, on n'en fout pas une rame... Mensonge ensuite parce que le statut même de "dramaturge-bien-connu-sur-les-scènes-internationales-RodrigoGarcia» l'inscrit en porte-à-faux du discours révolutionnaire que tiennent ses personnages. Le constat que fait Garcia de cette impuissance à faire coïncider la forme, en l'occurrence un discours apprécié par un public bourgeois et aisé, et le contenu, à savoir l'affirmation de l'hypocrisie de ce même public, a des effets sur le contenu de ses pièces. Conscient d'être un des dramaturges de la bourgeoisie occidentale, en dépit voire même à cause du discours provocateur qu'il met en scène, il ne parvient à assumer son statut qu'en redoublant de provocation, en exagérant l'idée que l'hypocrisie sociale gouverne le monde, et en nous contraignant à affronter d'un œil critique notre statut de spectateur en quête de divertissement.

\section{Une politique du mensonge}

Ce composé constant de mensonge et de vérité que nous propose Garcia s'assume comme un procédé théâtral à but politique. Mentir au théâtre trouve alors et son origine et sa raison d'être dans une position politique précise très critique envers le public, bourgeois par essence, qui vient voir ses pièces:

Je ne voulais plus travailler avec ce public cultivé que l'on croise dans les festivals. Toute cette consommation me semble toujours très en contradiction avec mon travail. Le fait que mes créations soient toujours beaucoup montrées devant un public européen qui ne me plaît pas tellement - au sens où il n'a pas vécu dans sa chair ce que d'autres subissent ailleurs - est une des raisons pour lesquelles j'ai fait des choses plus agressives, pour qu'il en prenne conscience, pour qu'il se sente mal. Mais cela ne rime à rien car au final le marché peut tout à fait m'assimiler. Du coup, j'ai l'impression d'être entretenu par un système qui intègre ma conception du théâtre au sein des phénomènes de la modernité (Garcia 2009: 22). 
Du fait de cette récupération inévitable, Garcia se sent comme englouti dans un jeu du «qui gagne, perd». Plus les pièces créées par le dramaturge sont appréciées par ce public qu'il aimerait désavouer, et plus il ressent ce triomphe comme un échec puisque ce succès signe son échec à irriter suffisamment le public pour qu'il siffle au lieu d'applaudir. Il évoque la "grande désillusion» qu'il a ressentie en comprenant que, aussi agressives que soient ces pièces, elles ne le seront jamais assez pour métamorphoser la représentation théâtrale de la révolution en une révolution sociale: "J'ai vécu un moment de grande désillusion en comprenant que je ne pouvais faire autre chose que de travailler pour une société de merde. Parce que je vis dans cette société de merde, de bienêtre, où tout est beau, magnifique, sans danger, sans menace.» (Garcia 2009: 23).

Or cette impression d'une récupération ou d'une absorption/digestion des morceaux pourtant les plus indigestes qu'il puisse livrer, explique qu'il clame haut et fort le mensonge du discours théâtral, y compris lorsque ce discours est celui de la dénonciation de l'hypocrisie sociale:

Des couches et des couches de mensonge par-dessus des mensonges. [...] La dénonciation est aussi un mensonge. Le voile est levé à ce qu'on dit pour que nous ayons accès à la vérité occulte, mais tu parles: le voile est levé pour que nous tombions sur un autre voile, un mensonge sous un autre mensonge sous un autre mensonge. (Garcia 2011: 25)

C'est dire que le théâtre ment y compris lorsqu'il dit la vérité, puisqu'en disant la vérité sur le public et le caractère méprisable de ses divertissements (théâtraux), il flatte ce public, qui peut tirer avantage de sa lucidité même et ressentir de la fierté à l'idée que, certes, il est égoïste, cupide... mais au moins, lui, le sait alors que les autres, qui ne vont pas au théâtre, l'ignorent. Garcia va même jusqu'à se moquer de l'hypocrisie de son propre discours: "Si tu vis comme tout le monde, jamais tu ne seras reconnu comme un artiste. Tu dois faire des choses bizarres. Ensuite il faut décider d'un style soit pour la défonce soit pour la déprime», (Garcia 2011: 25) étant entendu que, de toute façon, pour être apprécié du public, «il faut faire un choix. Question de marketing» (Garcia 10/2003: 33). Dans Golgotha Picnic, Garcia s'en prend aux sculptures d'Anish Kapoor, «ces fentes bleues, superbes, qui nous font oublier la douleur, qui se bornent à nous divertir et à décorer les espaces de douleur pour les dissimuler.» Or «ce qu'il faut faire, c'est tout le contraire: la présence réelle [...] qui n'a nul besoin de théâtralité, comme quand quelqu'un vous balance une vérité en face, pas un spectacle» (Garcia, 2003: 45).

Mais il va de soi que si la vérité est tout le contraire de la théâtralité, la pièce que l'on regarde n'est que «tout le contraire» de ce qu'il faudrait faire. Le mensonge dans ses pièces s'intègre à cet effort désespéré pour ne pas s'adresser au public auquel pourtant il s'adresse. Effort désespéré puisque, dans le cadre fermé et bourgeois de la situation théâtrale, la dénonciation et la provocation sont aussi des mensonges. Car n'est-ce pas un mensonge de faire croire que le discours révolutionnaire du théâtre peut aider à l'instauration d'une révolution alors que "c'est tout le contraire»? Si ce discours nous procure du 
plaisir, c'est probablement à l'inverse parce qu'il nous permet de faire l'économie de la révolution tout en nous confortant dans l'idée que nous avons en nous un fond révolutionnaire. Le dramaturge rejoint alors, dans la lucidité de son analyse sur le statut ambigu d'un théâtre provocateur, les critiques les plus traditionnelles adressées à la représentation théâtrale, qui, à l'instar de celles de Rousseau, reprochent au théâtre de produire l'inaction au lieu de l'action:

En donnant des pleurs à ces fictions, nous avons satisfait à tous les droits de l'humanité sans avoir plus rien à mettre du nôtre, au lieu que les infortunés en personne exigeraient de nous des soins dont nous sommes bien aises d'être exemptés. Au fond, quand un homme est allé pleurer des malheurs imaginaires, qu'a-t-on encore à exiger de lui ? Ne s'applaudit-il pas de sa belle âme? [...] Que voudrait-on qu'il fît de plus? Plus j'y réfléchis, et plus je trouve que tout ce qu'on met en représentation au théâtre, on ne l'approche pas de nous, on l'en éloigne. (Rousseau 1758: 78)

Et parallèlement, ne pourrait-on considérer qu'en soumettant au public d'un théâtre des discours révolutionnaires, on l'éloigne d'autant plus d'une action révolutionnaire? Et le spectateur ne se ment-il pas à lui-même sur les raisons de son amour du théâtre? Jean Baudrillard affirme ainsi que si nous regardons avec tant d'intérêt les informations saturées de bruit et de fureur, c'est paradoxalement parce qu'elles nous rassurent:

Quand nous regardons les images du monde, qui distinguera cette brève irruption de la réalité, du plaisir profond de n'y être pas? L'image, le message, tout ceci que nous consommons, c'est notre quiétude scellée par la distance au monde et que berce, plus qu'elle ne la compromet, l'allusion même violente au réel. (Baudrillard 1970: 33)

Nous avons besoin de consommer des représentations de la violence pour nous convaincre que notre vie calfeutrée vaut mieux que celle qui sévit à l'extérieur: «Il faut la violence et l'inhumanité du monde extérieur pour que non seulement la sécurité s'éprouve plus profondément comme telle, mais aussi soit justifiée de se choisir comme telle (Baudrillard 1970 : 34). La lucidité de Garcia devant cette fonction anti-révolutionnaire du théâtre, y compris d'un théâtre qui profère des discours révolutionnaires, explique que le dramaturge fasse du théâtre un lieu où sont proférés des mensonges.

\section{Claudio Tolcachir ou quand la vérité est dans le mensonge}

$\mathrm{Au}$ théâtre politique et misanthrope de Garcia qui dénonce, parfois agressivement, l'hypocrisie humaine, répond le théâtre intimiste et philanthrope de Claudio Tolcachir, qui nous permet de voir ses personnages en train de mentir pour mieux nous émouvoir de la nature humaine, en en montrant la vulnérabilité et la fragilité. De Garcia, on pourrait dire qu'il use du mensonge comme un moyen de créer la confusion entre ce qui est sincère et ce qui ne l'est pas dans un but très précis: refuser au public une position confortable. Il se situe ainsi à l'antithèse de Tolcachir qui cherche, à l'inverse, par le biais du 
mensonge, à accroître l'effet de vie du personnage, en le faisant passer du statut de personnage à celui de personne.

Chez Tolcachir, le mensonge, dissimulé jusqu'au bout par le menteur, ne se révèle comme tel que parce que le personnage est trahi par les faits à un moment donné : bien décidé à nous faire croire en la réalité de ces personnages, Tolcachir nous rend complice d'une scène où nous voyons dans un premier temps évoluer des êtres dans leur intimité. Puis dans un second temps, Tolcachir crée la surprise en nous montrant que, plus intime encore que les scènes de confidence qu'il nous a livrées, existe l'intimité dévoilée par le mensonge lui-même. Bref, autant chez Garcia, le mensonge tend à refuser au spectateur le droit de savoir, autant chez Tolcachir, le mensonge nous met au plus prêt de la vérité des êtres en nous introduisant dans leurs secrets de famille. Là même où, chez Garcia, le mensonge était un moyen de nous refuser toute complicité, il est chez Tolcachir une main tendue au public pour qu'il puisse pénétrer à l'intérieur même du psychisme des personnages.

De fait, Tercer Cuerpo. (L'histoire d'une tentative absurde) est l'histoire d'un mensonge ou plutôt d'une concentration, en un même espace, de menteurs. Dans la hiérarchie des mensonges les plus pathétiques, celui de Sandra, célibataire, occupe une place privilégiée: Sandra ment au médecin qu'elle va consulter en lui faisant croire que son mari, stérile, ne peut pas venir aux différentes consultations médicales que tous deux auraient sollicitées. Elle espère ainsi qu'il acceptera de pratiquer sur elle une fécondation artificielle sans qu'il juge utile la présence du mari. Et puis, devant la réticence du médecin, elle se voit contrainte de lui avouer:

Docteur, je dois vous dire quelque chose. C'est très embarrassant. Je vous ai menti. Je n'ai pas de mari. Vous vous en doutiez? Je n'en ai jamais eu. Enfin presque, un tout petit peu. [...] Et je ne peux plus attendre et je veux avoir un enfant. C'est ce que je désire le plus au monde. Je m'en fous de l'avoir toute seule. [...] Vous ne pouvez pas me faire un enfant? Je vous laisse les analyses. Et s'il vous plaît, ne le dites à personne. (Tolcachir 2011 : 39)

Mais Tolcachir ne s'est pas contenté de nous faire assister en spectateurs à cette mise à nu de l'intimité de Sandra : il a fait en sorte qu'il n'y ait pas d'acteur jouant le médecin. Le dialogue de Sandra n'est ainsi qu'un monologue où, faisant face au public, Sandra feint de s'adresser au docteur, personnage dont nous, spectateurs, sommes censés penser qu'il est là alors qu'il n'existe pas. Or, c'est bien à nous, spectateurs, que Sandra s'adresse, c'est à nous qu'elle ment, et c'est à nous encore qu'elle demande désespérément de lui faire un enfant... Le mensonge apparaît ainsi conjointement comme un acte sacrificiel et une demande d'amour: Sandra sacrifie sa sincérité pour être aimée parce qu'elle ne l'est pas, puis, face à son échec, elle sacrifie son intégrité en avouant son mensonge pour obtenir un objet d'amour et de désir: un enfant. Mais, parce qu'elle s'est construite sur la base du mensonge, la vérité que Sandra énonce enfin: "je ne peux plus attendre et je veux avoir un enfant» s'avère plus vraie qu'une vérité nue et qui ne se serait pas appuyée auparavant sur un mensonge. Car plus authentique encore que 
la révélation de son désir d'un enfant, le mensonge que Sandra, désespérée, se résout à avouer, fait de ce personnage pathétique un être si sincère et authentique qu'il en deviendrait presque réel.

Dans cette pièce, Sandra n'est pas la seule à mentir: Hector, un sexagénaire célibataire, ment à ses deux collègues de travail, en l'occurrence Sandra et Monica, parce qu'il ne parvient à leur avouer ni son homosexualité ni sa tentative absurde d'avoir une liaison avec un tout jeune homme. Et Monica ment à ses deux collègues, Sandra et Hector, en leur faisant croire qu'elle quitte, comme eux, le bureau le soir pour rentrer chez elle alors qu'en réalité elle n'a plus de logement et dort au bureau. Enfin Sandra ment à Hector et Monica, en leur faisant croire que son couple s'entend à merveille, alors qu'en réalité, elle n'a connu son mari que quelques mois avant qu'il ne l'abandonne. Or la volonté désespérée de mentir pour taire cette vie si inconsistante qu'ils en ont honte, va unir paradoxalement ces trois personnages autour d'un même secret: le secret de leur désespérance. Car ce sont bien les mensonges qu'ils s'adressent l'un l'autre qui, au lieu de les éloigner par la méfiance qu'ils devraient désormais se porter, rapprochent les trois personnages: comme si se créait un lien de pudeur, comme si le fait même qu'ils ressentent le besoin de se mentir l'un l'autre témoignait qu'ils tiennent l'un à l'autre, et qu'ils ont besoin de leur reconnaissance. Le mensonge de Sandra n'est que la face visible de la pudeur derrière laquelle elle veut cacher son mal-être et le vide de son existence. Nietzsche, juste avant de définir une forme d'amitié qu'il appelle du beau nom d'amitié stellaire, écrit: «Dans les contacts avec les personnes qui ont la pudeur des sentiments, il faut savoir dissimuler: elles sont susceptibles d'une haine subite pour qui surprend chez elles un sentiment délicat ou sublime, comme s'il avait vu leurs secrets" (Nietzsche 1882: 54). Et Nietzsche de fustiger la volonté de dire la vérité:

Ce mauvais goût, cette volonté de vérité, de la "vérité à tout prix», nous l'avons désormais en exécration: nous sommes trop aguerris, trop profonds pour cela... Nous ne croyons plus que la vérité soit encore la vérité dès qu'on lui retire ses voiles. Aujourd'hui c'est pour nous une affaire de convenance qu'on ne saurait voir toute chose mise à nu, ni vouloir tout «savoir». (Nietzsche 1882:19)

Paradoxalement, le mensonge des trois personnages de Tercer Cuerpo est tout à leur honneur, d'une part parce qu'il témoigne d'une certaine humilité, d'aucuns diraient d'une forme de lucidité face à la médiocrité de leurs vies, et d'autre part parce qu'il s'avère être une tentative pour être aimé. Sans leur mensonge, Sandra, Monica et Hector seraient pitoyables et pathétiques certes, mais médiocres et sans épaisseur. Le mensonge en revanche les transfigure: il fait ressortir leur sensibilité pudique et leur désir d'amour. Quant au secret partagé, il explique en partie le plaisir ressenti lors d'une représentation d'une pièce de Tolcachir :

Il y a toujours une aristocratie du secret. D'avoir vu ensemble une chose très rare, de détenir ensemble quelque secret accablant [...] crée entre deux êtres une fraternité durable, une sorte d'entente non concertée qui ne se discute pas. (Jankélévitch 1979: 49) 
L'exhibition du mensonge permet d'accroître le plaisir du spectateur en exacerbant son voyeurisme. Vincent Jouve constate ainsi que «l'intimité passionnelle est aussi passionnante que l'intimité corporelle» (Jouve 1992 : 157). Or c'est bien l'intimité passionnelle de ces trois personnes en mal d'amour que nous livre Tolcachir et qui nous procure tant de plaisir. Établir un parallèle avec la photographie de Doisneau, Le regard oblique, et le commentaire qu'en livre Philippe Hamon, se révèle éclairant. Devant la vitrine d'un galeriste, une femme montre à son mari une œuvre dont on imagine sans peine qu'elle n'a rien de choquant ni de novateur. Absorbée par cette contemplation, elle lui livre ses impressions sans se douter que l'intérêt de l'époux se porte ailleurs et que son regard, courbe ou fourbe, s'est fourvoyé et a obliqué vers un autre tableau, représentant une jeune femme à l'attitude provocante. Nonchalamment accoudée, de dos, nue à l'exception de longues bottes noires, la jeune femme du tableau contraste singulièrement avec la femme guindée qui accompagne son mari. Philippe Hamon en fait le commentaire suivant:

Moi, spectateur de la photo de Doisneau, je vois que la femme habillée ne voit pas que l'homme regarde la femme nue. Ce que le jugement extérieur, hors de la scène, me permet de voir, c'est le spectacle d'un aveuglement. [...] Regardeurvoyeur (ob-scène) de la photo, mis en recul critique par rapport à la scène, sollicité aussi dans son regard oblique (sur la femme nue) qu'il peut, lui, regarder tout à loisir et en toute impunité, le spectateur se croit en position d'omniscience. (Hamon 1996: 12)

Le cliché de Doisneau nous permet de saisir l'origine du plaisir que procure le spectacle du mensonge au théâtre: moi, spectateur/voyeur, j'ai le plaisir de voir que ce mari bourgeois est un menteur, que sa fidélité affichée est démentie par l'infidélité de son regard. Et moi, spectateur/jouisseur, je vois que cette femme bourgeoise ne voit pas que son mari lui ment. On comprend bien l'origine du plaisir au spectacle: la connaissance qu'un des personnages ment instaure une supériorité du spectateur sur le menteur.

Le dévoilement du mensonge introduit le public au plus près des personnages, le rendant complice d'un viol d'intimité. Un tel mensonge prend place à l'intérieur d'une mise en scènequasi-sacrificielle où le dramaturge fait don de ses personnages au spectateur: affaibli, mis à nu par l'aveu de son mensonge, le personnage nous est livré dans son intégralité. La demande de Sandra au spectateur/médecin "vous ne voulez pas me faire un enfant? "vaut alors comme un don de sa personne. Le mensonge s'intègre alors à une mise en scène où les cloisons sont abattues, et où la scène, sans estrade, n'est ni plus ni moins éclairée que la salle. Pour accentuer encore cette absence d'intimité, Tolcachir, metteur en scène de ses propres pièces, a fait de l'espace un espace total: à la fois restaurant, bureau, maison, cabinet médical et coulisses, puisque les acteurs ne quittent jamais la scène même lorsqu'ils ne jouent pas. Par cette fusion, Tolcachir parvient à construire une aire de jeu propice au mensonge: l'absence d'intimité suscite le mensonge. Ainsi, lorsque Monica est découverte par ses deux collègues dormant en chemise de nuit le matin dans le bureau, elle sollicite 
leur pitié: «Je vous demande pardon. [...] Je n’ai pas d’intimité ici.» (Tolcachir 2011 : 42). Les théâtres de l'intime et, en leur sein, tout ce qui concerne l'intimité du désir... désir d'un enfant pour Sandra, désir d'un homme pour Hector, désir d'un chez-soi pour Monica convoquent le mensonge comme élément majeur du discours et, ce faisant, parviennent à dénuder un morceau de l'âme humaine.

Aussi différents que soient les théâtres de Tolcachir et de Garcia, ils expriment tous deux tant la vérité du mensonge que le mensonge de la vérité: l'oscillation permanente entre la franchise qui semble émaner de la voix de Garcia et les mensonges qu'il dit proférer nous rappelle que les relations humaines, toujours évolutives, se situent en-deçà du vrai et du faux. Et de même en est-il de Tolcachir lorsqu'il montre combien le mensonge d'un individu peut révéler la vérité sur lui, et combien cette vérité parfois n'est autre qu'un désir de mentir pour ne pas se dévoiler. Tous deux nous disent ainsi à leur manière ce dont Sartre déjà nous prévenait dans Les Mots:

Ce que je viens d'écrire est faux. Vrai. Ni vrai ni faux comme tout ce que l'on écrit sur les fous, sur les hommes [...]. J'ai vu par la suite qu'on pouvait tout connaître de nos affections hormis leur force, c'est-à-dire leur sincérité.» (Sartre 1964 : 60)

\section{Bibliographie}

Baudrillard J., 1970, La société de consommation, Paris, Gallimard Tel.

Garcia R., 10/2003, Jardinage Humain, Besançon, Les Solitaires intempestifs.

Garcia R., 11/2003, Roi Lear, Besançon, Les Solitaires intempestifs.

Garcia R., 2009, Bleue, saignante, à point, carbonisée, Besançon, Les Solitaires intempestifs.

Garcia R., 2011, Golgotha Picnic, Besançon, Les Solitaires intempestifs.

Hamon P., 1996, L'Ironie littéraire, Paris, Hachette Littératures.

Jankélévitch V., 1979, L'Ironie, Paris, Champs Flammarion.

Jouve V., 1992, L'Effet personnage dans le roman, Paris, PUF Écriture.

Liddell A., 2011, Maudit soit l'homme qui se confie à l'homme, Besançon, Les Solitaires intempestifs.

Nietzsche F., (1882), 1950, Le Gai Savoir, Paris, Gallimard Idées.

Py O., Le Soleil, 2011, Paris, Actes Sud Papiers.

Rousseau J.-J., (1758), 1967, Lettre à d'Alembert, Paris, Garnier Flammarion.

Sartre J.-P., 1943, L'Être et le néant, Paris, Gallimard Tel.

Sartre J.-P., 1964, Les Mots, Paris, Gallimard Folio Essais.

Shakespeare W. (1603), 1978, Hamlet, Paris, Gallimard Folio.

Tolcachir C., 2011, L'histoire d'une tentative absurde, L'île Saint Denis, Voix navigables Editions. 